\title{
PKMYT1 regulates the proliferation and epithelial-mesenchymal transition of oral squamous cell carcinoma cells by targeting $\mathrm{CCNA} 2$
}

\author{
YE CAI and WEIDONG YANG \\ Department of Endodontics, Nanjing Stomatological Hospital, \\ Medical School of Nanjing University, Nanjing, Jiangsu 210008, P.R. China
}

Received September 9, 2021; Accepted November 8, 2021

DOI: $10.3892 / \mathrm{ol} .2021 .13181$

\begin{abstract}
Oral squamous cell carcinoma (OSCC) has gradually become a global public health issue in recent years. Therefore, the current study aimed to explore the mechanism of OSCC development and to identify a potential target that may be used in its treatment. The expression of protein kinase, membrane-associated tyrosine/threonine 1 (PKMYT1) and cyclin A2 (CCNA2) in SCC-9 cells was determined prior to and following transfection with short hairpin RNA targeting PKMYT1. Cell proliferation, colony-forming ability, migration and invasion were determined using Cell Counting Kit-8, colony formation, wound healing and Transwell assays, respectively. Furthermore, the expression of epithelial-mesenchymal transition (EMT)- and migration-related proteins were evaluated using western blot analysis. Additionally, co-immunoprecipitation was used to verify the binding of PKMYT1 and CCNA2. The results revealed that PKMYT1 was highly expressed in OSCC cells and that PKMYT1 knockdown could inhibit proliferation, colony formation, migration, invasion, EMT and CCNA2 expression in SCC-9 cells. In addition, PKMYT1 was demonstrated to bind to CCNA2, and knocking down PKMYT1 resulted in inhibitory effects on cell proliferation, colony formation ability, migration, invasion and EMT by downregulating CCNA2 expression. PKMYT1 was observed to regulate the proliferation, migration and EMT of OSCC cells by targeting CCNA2, which may be used in the future to improve OSCC treatment.
\end{abstract}

Correspondence to: Dr Weidong Yang, Department of Endodontics, Nanjing Stomatological Hospital, Medical School of Nanjing University, 30 Zhongyang Road, Nanjing, Jiangsu 210008, P.R. China

E-mail: yangweidong45@163.com

Key words: oral squamous cell carcinoma, protein kinase, membrane-associated tyrosine/threonine 1, cyclin A2, proliferation, migration, epithelial-mesenchymal transition

\section{Introduction}

Oral squamous cell carcinoma (OSCC) is one of the most common malignancies of the head and neck, accounting for $>90 \%$ of all oral cancer cases, and is the sixth most common type of cancer globally (1). Currently, the management of patients with OSCC is based on histological parameters, such as TNM stage and tumor grade (2). If there is no cervical lymph node metastasis in patients with early-stage oral cancer, surgery or radiotherapy alone has been observed to exert a beneficial effect, whereas surgery combined with radiotherapy is a more suitable treatment for intermediate- or advanced-stage oral cancer (3). Long-term addiction to alcohol and tobacco, poor oral hygiene, long-term irritation by foreign bodies and malnutrition, particularly insufficient vitamin A levels, have been indicated to cause OSCC (4). The complex occurrence and development of oral cancer is regulated at the genetic level, and protein expression is regulated at the transcriptional and translational levels $(5,6)$. The analysis and use of valuable biomarkers that are primarily associated with the pathogenesis of OSCC can provide a more comprehensive and detailed understanding of the disease, which may lead to the development of targeted, personalized, practical and effective oral cancer treatment options (7). Therefore, to improve the survival rate and quality of life of patients with OSCC, effective tumor-related factors, novel therapeutic methods and antitumor drugs must be urgently identified.

Protein kinase, membrane-associated tyrosine/threonine 1 (PKMYT1) is located at 16p13.3 on human chromosome 16, and encodes an important protein belonging to the WEE kinase family $(8,9)$. PKMYT1 is responsible for encoding a member of the serine/threonine protein kinase family, and the encoded protein negatively regulates the $G_{2} / M$ transition of the cell cycle via phosphorylation and inactivation of cyclin-dependent kinase 1 (10). An increasing number of studies have indicated that PKMYT1 overexpression promotes cell proliferation, migration, invasion, colony-forming ability and epithelial-mesenchymal transition (EMT) in multiple tumor types (11). For example, PKMYT1 has previously been revealed to be upregulated in breast cancer, and was also indicated to regulate cell proliferation by maintaining the cell cycle and genomic stability (12). It has also been revealed that 
PKMYT1 may promote cell proliferation, invasion and migration in ovarian cancer by targeting sirtuin 3 (13). Additionally, PKMYT1 has been indicated to promote cell proliferation and apoptosis resistance in gastric cancer cells by activating the MAPK signaling pathway (8). Although PKMYT1 has been studied in various cancer types, the role of PKMYT1 in OSCC remains to be investigated.

The hypothesis that PKMYT1 also serves a role in oral cancer has been previously proposed. According to the Gene Expression Omnibus (GEO) database (https://www.ncbi.nlm. nih.gov/geo/), PKMYT1 is highly expressed in OSCC tissues (GSE37991). Therefore, the present study aimed to explore the role of PKMYT1 in OSCC, and its target was predicted using bioinformatics analysis. The results of the current study may provide useful information on the mechanisms of cell proliferation, migration and the EMT process in OSCC, and may facilitate the identification of a novel target that may be used in the treatment of this disease.

\section{Materials and methods}

Cell culture and transfection. Human oral keratinocytes (HOK-16B), human tongue squamous cell carcinoma (CAL-27, HSC-4 and SCC-9) cells were purchased from BeNa Culture Collection (Beijing Beina Chunglian Institute of Biotechnology) and were cultured in DMEM (Gibco; Thermo Fisher Scientific, Inc.) supplemented with 10\% FBS (Gibco; Thermo Fisher Scientific, Inc.) at $37^{\circ} \mathrm{C}$ in a humidified atmosphere with $5 \% \mathrm{CO}_{2}$.

The pcDNA3.1-cyclin A2 (CCNA2) and empty pcDNA3.1 vectors, short hairpin RNA (shRNA) targeting PKMYT1 (shRNA-PKMYT1-1, target sequence: 5'-CTA TGCGGTAAAGCGTTCCAT-3' and shRNA-PKMYT1-2, target sequence: 5'-GCTGCGTTCTGTCCTTGTCAT-3') and a nonspecific sequence used as a negative control (NC; target sequence: 5'-CAACAAGATGAAGAGCACCAA-3') were purchased from Shanghai GenePharma Co., Ltd. SCC-9 cells $\left(5 \times 10^{5}\right.$ cells/well) were seeded into 6 -well plates and transfected with $10 \mathrm{nM}$ pcDNA plasmid or shRNA using Lipofectamine $^{\circledR} 3000$ (Invitrogen; Thermo Fisher Scientific, Inc.) according to the manufacturer's protocol. Following $6 \mathrm{~h}$ of transfection, the transfection reagent in the wells were replaced with fresh DMEM supplemented with 10\% FBS and cells were cultured for 2 days (all from Gibco; Thermo Fisher Scientific, Inc.).

Cell Counting Kit-8 (CCK-8) assay. SCC-9 cells $\left(1 \times 10^{3}\right.$ cells/well) were seeded into $96-$ well plates and incubated for $24 \mathrm{~h}$. Following 24, 48 and $72 \mathrm{~h}$ of incubation, $10 \mu \mathrm{l} \mathrm{CCK}-8$ solution (Sigma-Aldrich; Merck KGaA) was added to each well, and the cells were incubated at $37^{\circ} \mathrm{C}$ for an additional $1 \mathrm{~h}$. Finally, the optical density was determined at $450 \mathrm{~nm}$ using a microplate reader (Thermo Fisher Scientific, Inc.).

Colony formation assay. SCC- 9 cells $\left(3 \times 10^{3}\right.$ cells/well) were seeded into 6 -well plates and incubated for 14 days at $37^{\circ} \mathrm{C}$. The colonies were then fixed with $4 \%$ methanol at room temperature for $15 \mathrm{~min}$, and next stained using $0.1 \%$ crystal violet at room temperature for $15 \mathrm{~min}$. Visible colonies of $>50$ cells were observed using an inverted microscope (magnification, x100; Olympus Corporation) and analyzed using ImageJ software (v1.8; National Institutes of Health).

Wound healing assay. SCC-9 cells ( $5 \times 10^{5}$ cells/well) were inoculated in 6-well plates and incubated until the reaching $\sim 90 \%$ confluence. A $200-\mu 1$ sterile pipette tip was applied to create a wound in the cell monolayer. Following washing with PBS, the cells were cultured in serum-free DMEM (Gibco; Thermo Fisher Scientific, Inc.) at $37^{\circ} \mathrm{C}$ for 24 h. Images were captured at 0 and $48 \mathrm{~h}$ under an inverted microscope (magnification, x100; Olympus Corporation).

Transwell assay. The cell invasion assay was performed using Transwell chambers ( $8 \mu \mathrm{m}$ pore size; Corning, Inc.). SCC-9 cells $\left(3 \times 10^{4}\right.$ cells) were suspended in $1 \mathrm{ml}$ serum-free DMEM. The upper chamber was pre-coated with $50 \mu$ l Matrigel (Sigma-Aldrich; Merck KGaA) with serum-free DMEM (1:8 diluted) to form a gel at $37^{\circ} \mathrm{C}$ for $30 \mathrm{~min}$, and $0.1 \mathrm{ml}$ cell suspension was added to each well of the upper chamber. The lower chamber was filled with DMEM supplemented with $20 \%$ FBS. Following $24 \mathrm{~h}$ of incubation at $37^{\circ} \mathrm{C}$, the cells in the lower chamber were collected, fixed using $4 \%$ paraformaldehyde at room temperature for $10 \mathrm{~min}$ and stained using $0.5 \%$ crystal violet solution at room temperature for $10 \mathrm{~min}$ (Sigma-Aldrich; Merck KGaA). Finally, the stained cells were counted using an inverted microscope (magnification, x100; Olympus Corporation).

Co-immunoprecipitation (co-IP) assay. Co-IP assay was performed to verify the binding between PKMYT1 and CCNA2. The isolation and quantification of proteins from SCC-9 cells was conducted using RIPA lysis buffer (Beijing Solarbio Science \& Technology Co., Ltd.) and BCA kits (Beyotime Institute of Biotechnology), respectively. For IP, $2 \mu \mathrm{g}$ antibodies against PKMYT1 (1:100; cat. no. H00009088-D01; Abnova) or CCNA2 (1:100; cat. no. 67955; Cell Signaling Technology, Inc.) were combined with $500 \mu \mathrm{g}$ proteins and incubated overnight at $4^{\circ} \mathrm{C}$. Subsequently, $40 \mu 1$ protein G/A agarose beads (Invitrogen; Thermo Fisher Scientific, Inc.) were added to the solution and incubated with the cell lysates for $2 \mathrm{~h}$. Subsequently, the precipitated proteins were re-suspended in 2X SDS-PAGE loading buffer (Beyotime Institute of Biotechnology), boiled for $5 \mathrm{~min}$ and eluted from the beads. Finally, the protein complexes were determined using western blot analysis as described below.

Reverse transcription-quantitative PCR (RT-qPCR). Total RNA was collected from SCC-9 cells using TRIzol ${ }^{\circledR}$ reagent (Thermo Fisher Scientific, Inc.) and placed on ice for $15 \mathrm{~min}$. PrimeScript $^{\mathrm{TM}}$ Reverse Transcription Reagent Kit (Takara Bio, Inc.) was used to reverse transcribe RNA into cDNA according to the manufacturer's protocol. Subsequently, QuantiNova SYBR-Green PCR Kit (Qiagen AB) was employed according to the manufacturer's protocol for qPCR in conjunction with an ABI 7500 System (Thermo Fisher Scientific, Inc.). The $2^{-\Delta \Delta \mathrm{Cq}}$ method (14) was used for analysis of the results (14). GAPDH was used as a control for normalization. The primers used were as follows: PKMYT1 forward, 5'-CATGGCTCCTACGGAGAGGT-3' and reverse, 5'-ACA TGGAACGCTTTACCGCAT-3'; and GAPDH forward, 
5'-GGGTGTGAACCATGAGAAGT-3' and reverse, 5'-GGC ATGGACTGTGGTCATGA-3'.

Western blot analysis. Total proteins from SCC-9 cells were extracted using RIPA lysis buffer (Beijing Solarbio Science $\&$ Technology Co., Ltd.) and quantified using a BCA kit (Beyotime Institute of Biotechnology). After protein denaturation by boiling at $100^{\circ} \mathrm{C}$ for $8 \mathrm{~min}, 12 \%$ SDS-PAGE was performed. Upon transfer onto PVDF membranes, the membranes were blocked in 5\% fat-free milk for $2 \mathrm{~h}$ at room temperature, washed with TBS twice at room temperature (5 sec per wash), and incubated with primary antibodies (all from Abcam) at $4^{\circ} \mathrm{C}$ overnight. The primary antibodies used were as follows: Anti-PKMYT1 (1:500; cat. no. ab200387), anti-MMP2 (1:1,000; cat. no. ab92536), anti-MMP9 (1:1,000; cat.no. ab283575), anti-E-cadherin (1:10,000; cat. no. ab40772), anti-N-cadherin (1:5,000; cat. no. ab76011), anti-Snail (1:1,000; cat. no. ab216347), anti-zinc finger E-box binding homeobox 1 (ZEB1; 1:500; cat. no. ab203829), anti-CCNA2 (1:2,000; cat. no. ab181591) and GAPDH (1:10,000; cat. no. ab181602). The next day, the membranes were washed with TBS- $0.05 \%$ Tween-20 thrice at room temperature (5 min per wash), and then incubated with an IgG-horseradish peroxidase-conjugated goat anti-rabbit secondary antibody $(1: 10,000$; cat. no. ab6721; Abcam) for $1 \mathrm{~h}$ at room temperature. Finally, protein bands were detected using an ECL kit (Beyotime Institute of Biotechnology), and the results were normalized to GAPDH and analyzed using ImageJ software (v1.8; National Institutes of Health).

Bioinformatics and statistical analysis. The Search Tool for the Retrieval of Interacting Genes/Proteins (STRING; http://www. string-db.org/) (15) and GeneMANIA (http://genemania. org/) (16) online databases were used to search associations between known proteins. All experiments were repeated at least three times independently, and the results were expressed as the mean \pm standard deviation. Statistical analysis was performed using SPSS 19.0 software (IBM Corp.). One-way ANOVA followed by Tukey's post hoc test was used to evaluate the statistical significance of the results. $\mathrm{P}<0.05$ was considered to indicate a statistically significant difference.

\section{Results}

PKMYT1 is upregulated in OSCC cells, and PKMYT1 knockdown inhibits the proliferation and colony formation of SCC-9 cells. The relative mRNA and protein expression of PKMYT1 in normal human HOK-16B and OSCC cells, including CAL-27, HSC-4 and SCC-9 cells, was detected using RT-qPCR and western blot analyses. The results revealed that the relative mRNA and protein expression of PKMYT1 in OSCC cells was significantly upregulated compared with that of the HOK-16B group, particularly in SCC-9 cells (Fig. 1A and B). Therefore, SCC-9 cells were selected for the subsequent experiments.

To investigate the effects of PKMYT1 on OSCC cells, SCC-9 cells were transfected with sh-PKMYT1. As presented in Fig. 1C and D, PKMYT1 mRNA and protein expression was decreased in the sh-PKMYT1 group compared with that of the shRNA (NC) group, particularly in the sh-PKMYT1-1 group. Therefore, SCC-9 cells transfected with sh-PKMYT1-1 were used for subsequent experiments.

Cell proliferation was determined using a CCK-8 assay. As presented in Fig. 1E, the proliferation of SCC-9 cells was decreased following transfection with shRNA targeting PKMYT1. It was also revealed that PKMYT1 knockdown could significantly inhibit SCC-9 cell proliferation at the time points of 24, 48 and $72 \mathrm{~h}$. Furthermore, the colony-forming ability of SCC-9 cells was also found to be suppressed by PKMYT1 knockdown. These results revealed that PKMYT1 knockdown could inhibit the proliferation and colony-forming ability of SCC-9 cells.

PKMYT1 knockdown inhibits the migration, invasion and EMT of SCC-9 cells. The relative migration rate and invasion ability were assessed using wound healing and Transwell assays, respectively. The results presented in Fig. 2A showed that PKMYT1 knockdown suppressed the migration rate of SCC-9 cells compared with that of the shRNA NC group. The results also demonstrated that PKMYT1 knockdown exerted the same inhibitory effects on SCC-9 cell invasiveness, which was indicated by the decrease in the relative cell invasion rate (Fig. 2B).

Furthermore, the expression of MMP2 and MMP9, which was determined using western blot analysis, was markedly decreased by PKMYT1 knockdown (Fig. 2C). Additionally, the expression of EMT-related proteins was detected using western blot analysis. As presented in Fig. 2D, PKMYT1 knockdown significantly promoted E-cadherin expression and downregulated the expression of N-cadherin, Snail and ZEB1.

PKMYT1 knockdown inhibits the expression of CCNA2 in OSCC cells. The STRING and GeneMANIA databases suggested an association between PKMYT1 and CCNA2 (Fig. 3A). The mRNA and protein expression of CCNA2 in normal human HOK-16B cells and in OSCC cells, including CAL-27, HSC-4 and SCC-9 cells, was determined using RT-qPCR and western blot analyses, respectively. The results presented in Fig. 3B and $\mathrm{C}$ revealed that there was higher expression of CCNA2 in OSCC cells, particularly in SCC-9 cells, than in HOK-16B cells.

Considering the positive association between PKMYT1 and CCNA2, a co-IP assay was used to further analyze the binding of PKMYT1 to CCNA2. As presented in Fig. 3D, PKMYT1 was expressed in the anti-CCNA2 group and CCNA2 was expressed in the anti-PKMYT1 group, while IgG expression was not detected, revealing that PKMYT1 may combine with CCNA2. Compared with that of the shRNA $\mathrm{NC}$ group, the expression of CCNA2 was decreased in the sh-PKMYT1 group (Fig. 3E).

PKMYT1 knockdown inhibits the proliferation and colony formation of OSCC cells by downregulating CCNA2 expression. SCC-9 cells were transfected with pcDNA-CCNA2, and the expression of CCNA2 was subsequently determined using western blot analysis. The results demonstrated that the relative protein expression of CCNA2 in SCC-9 cells was upregulated in comparison with that of the pcDNA group (Fig. 3F). As is shown Fig. 3G and H, the decreased cell proliferation and colony formation caused by 
A

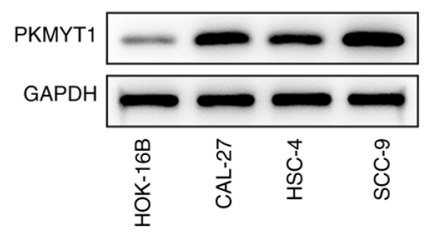

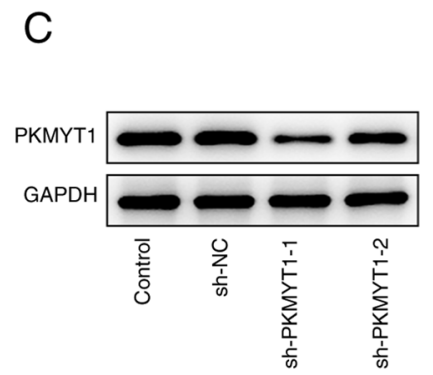
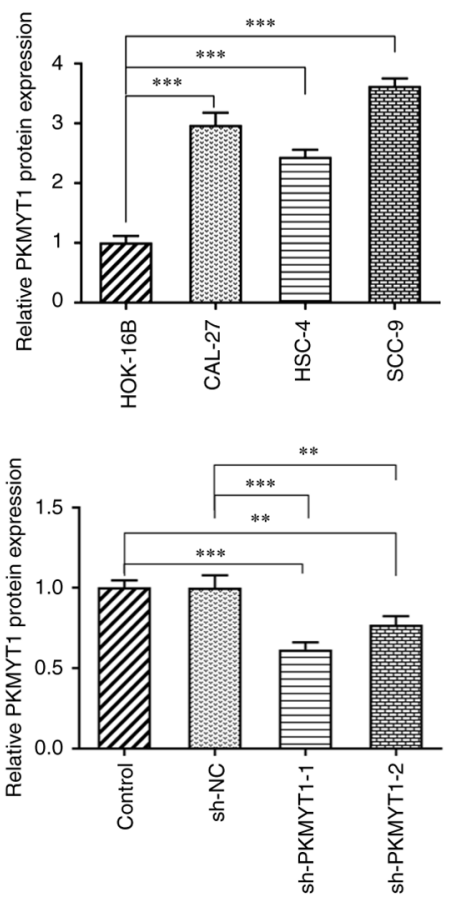

E

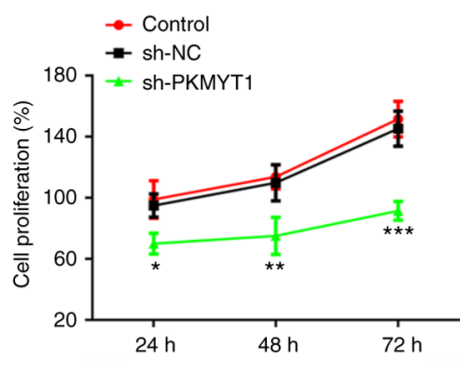

B

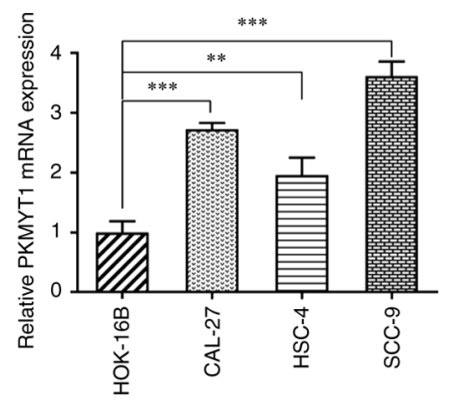

$\mathrm{D}$

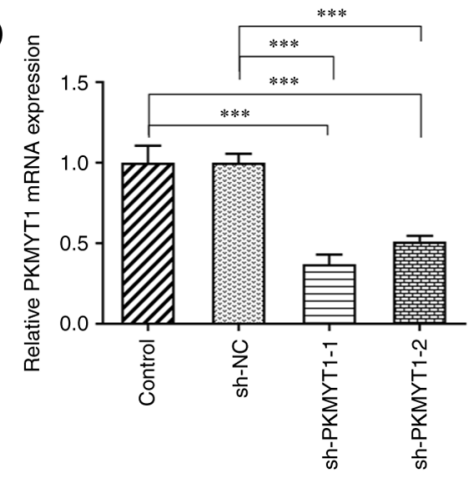

$\mathrm{F}$

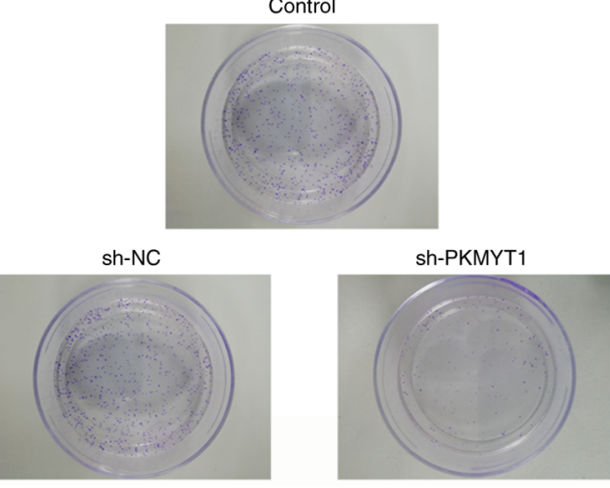

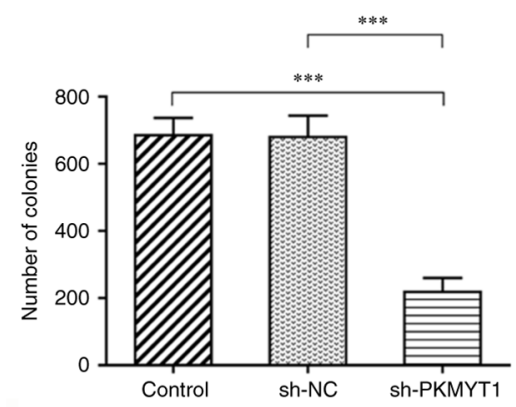

Figure 1. PKMYT1 is upregulated in SCC-9 cells, and its knockdown inhibits cell proliferation. (A) PKMYT1 protein expression was detected using western blot analysis. (B) PKMYT1 mRNA expression was detected using RT-qPCR. (C and D) Relative protein and mRNA expression levels were detected using (C) western blot analysis and (D) RT-qPCR, respectively. Cell proliferation was detected using (E) Cell Counting Kit- 8 . ${ }^{*} \mathrm{P}<0.05$, ${ }^{* * *} \mathrm{P}<0.01,{ }^{* * * *} \mathrm{P}<0.001$ vs. sh-NC. (F) Cell colony formation was determined using colony formation assays. ${ }^{* *} \mathrm{P}<0.01,{ }^{* * * *} \mathrm{P}<0.001$. PKMYT1, protein kinase, membrane-associated tyrosine/threonine 1; RT-qPCR, reverse transcription-quantitative PCR; sh-NC, negative control short hairpin RNA.

PKMYT1 knockdown was partially reversed by CCNA2 overexpression, suggesting that CCNA2 overexpression may partly abolish the inhibitory effects of PKMYT1 knockdown on SCC-9 cell proliferation and colony formation.

PKMYT1 knockdown inhibits the migration, invasion and EMT of SCC-9 cells by downregulating CCNA2 expression. Compared with those of the sh-PKMYT1 + pcDNA group, the decreased migration and invasion rates of SCC-9 cells were increased following CCNA2 overexpression (Fig. 4A and B).

Furthermore, the expression of MMP2 and MMP9, which was measured using western blot analysis, was partly increased in the sh-PKMYT1 + pcDNA-CCNA2 compared with that in the sh-PKMYT1 + pcDNA group (Fig. 4C). Additionally, as presented in Fig. 4D, CCNA2 overexpression partly inhibited the expression of E-cadherin, and increased the expression of $\mathrm{N}$-cadherin, Snail and ZEB1 in SCC-9 cells compared with the findings in the sh-PKMYT1 + pcDNA group.

\section{Discussion}

OSCC is one of the most aggressive tumors worldwide, and there is currently no optimal treatment for this disease (17). PKMYT1 is closely associated with tumor radiosensitivity, making it a candidate target for the development of improved treatments for OSCC (18). Previously, PKMYT1 inhibition has been reported to significantly suppress the proliferation of prostate cancer cells, thus serving as a novel therapeutic target for prostate cancer (19). Furthermore, Jeong et al (20) have suggested that PKMYT1 serves a vital oncogenic role in colorectal cancer, which is demonstrated by the increased proliferation, migration, invasion and colony forming ability of colorectal cancer cell lines. According to the GEO database, PKMYT1 was found to be upregulated in patients with OSCC (21). The results of the present study revealed that PKMYT1 was highly expressed in three OSCC cell lines compared with its expression in HOK-16B cells. The 
A
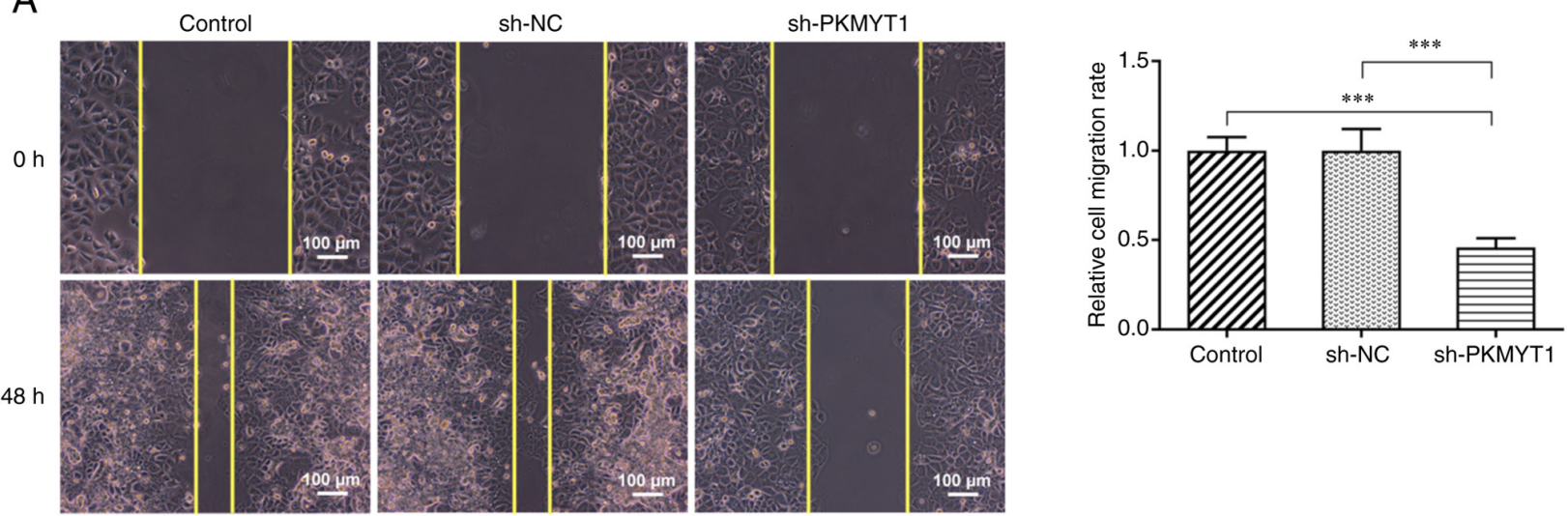

B Control sh-NC sh-PKMYT1
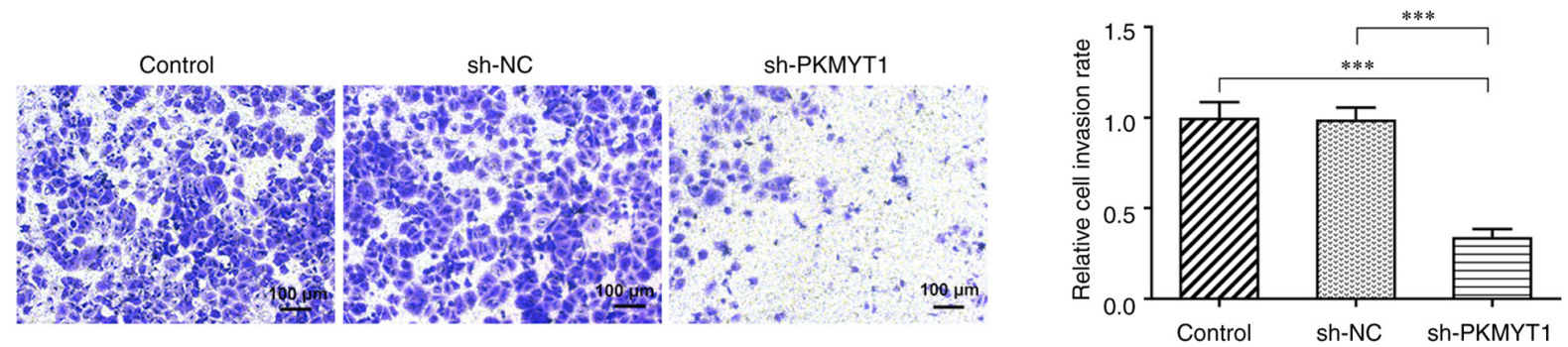

C
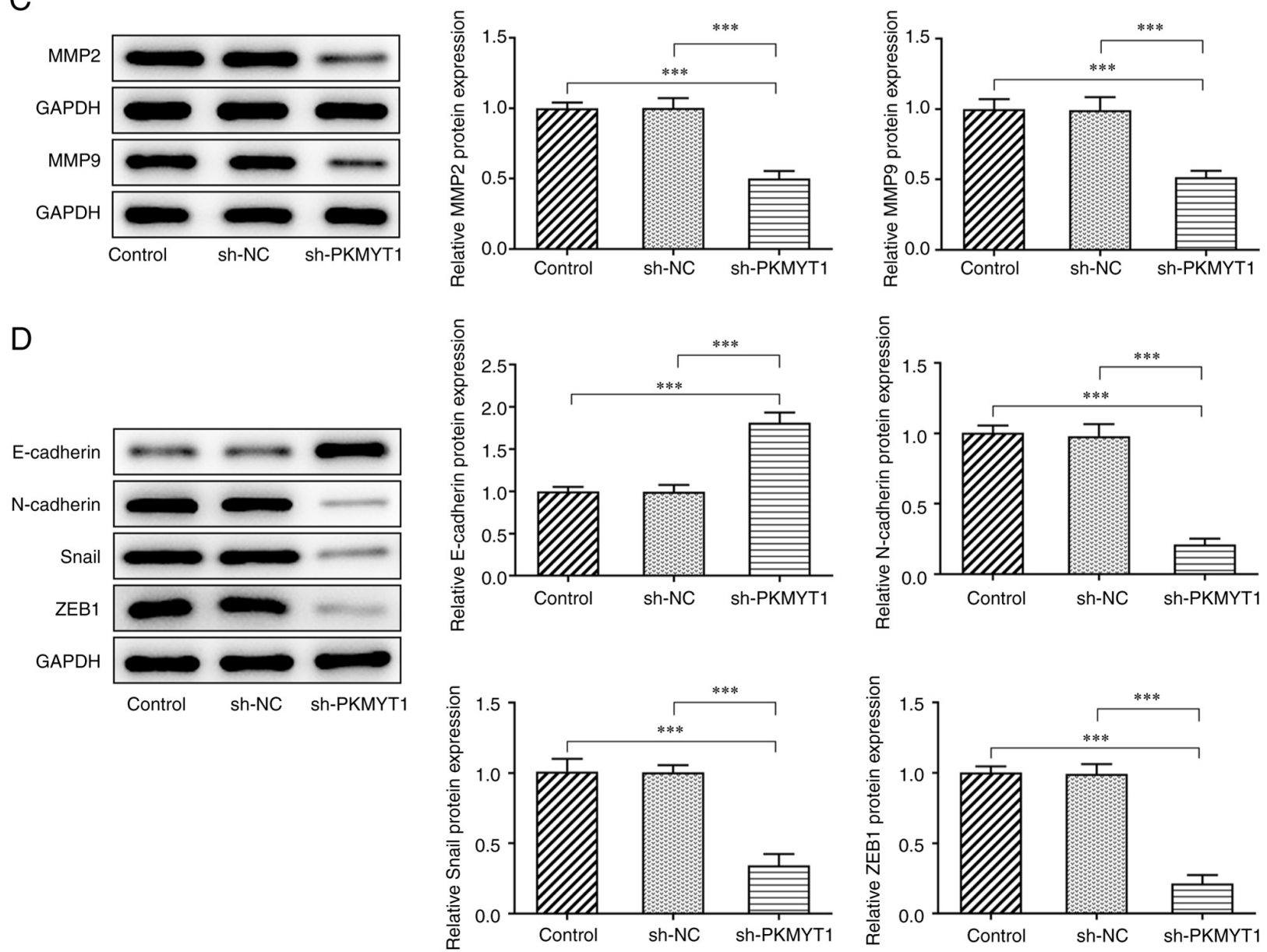

Figure 2. PKMYT1 knockdown inhibits the migration, invasion and EMT of SCC-9 cells. (A) Relative cell migration rate was detected using a wound healing assay. (B) Relative cell invasion rate was detected using a Transwell assay. (C) MMP2 and MMP9 expression levels were detected using western blot analysis. (D) The expression of EMT-related proteins was detected using western blot analysis. ${ }^{* * *} \mathrm{P}<0.001$. PKMYT1, protein kinase, membrane-associated tyrosine/threonine 1; EMT, epithelial-mesenchymal transition; sh-NC, negative control short hairpin RNA; ZEB1, zinc finger E-box binding homeobox 1.

expression level of PKMYT1 in HSC-4 cells was lower than that in other OSCC cell lines, which may be due to its non-metastatic nature (22). In addition, the expression levels of PKMYT1 in CAL-27 cells were higher than those in HSC-4 
A

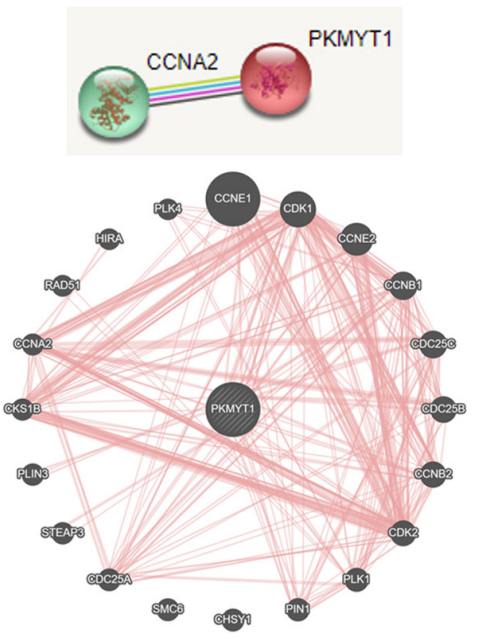

D
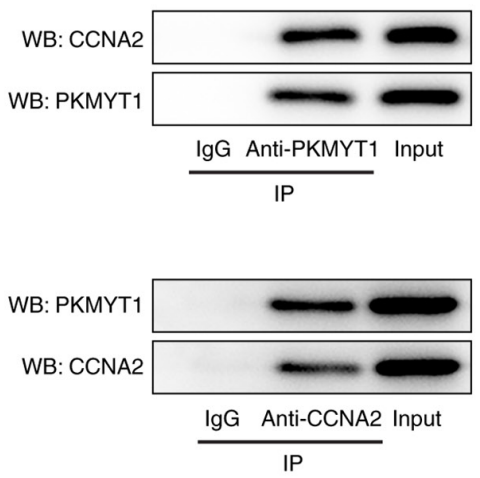

G

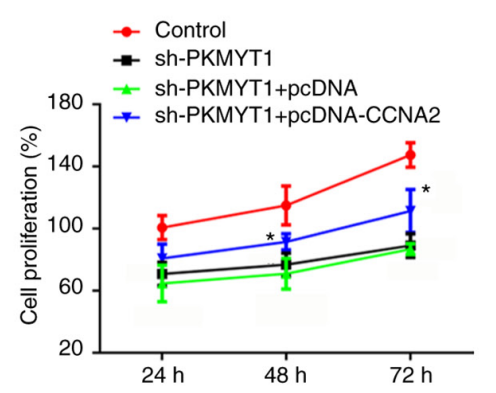

B

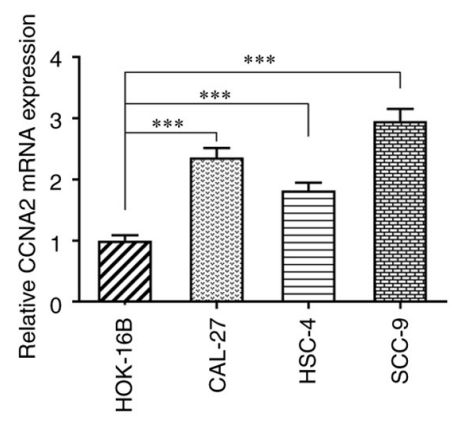

C
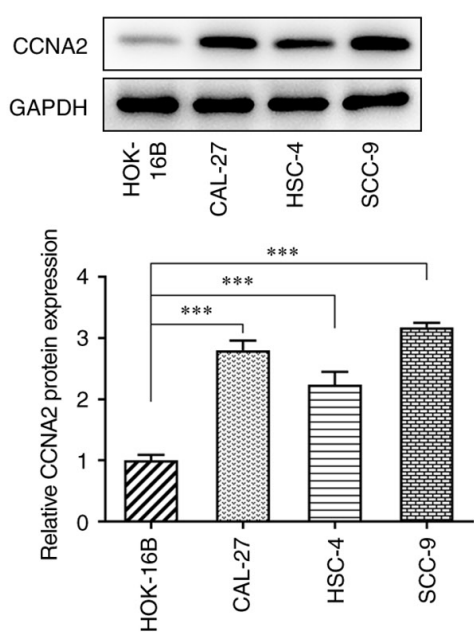

$\mathrm{E}$
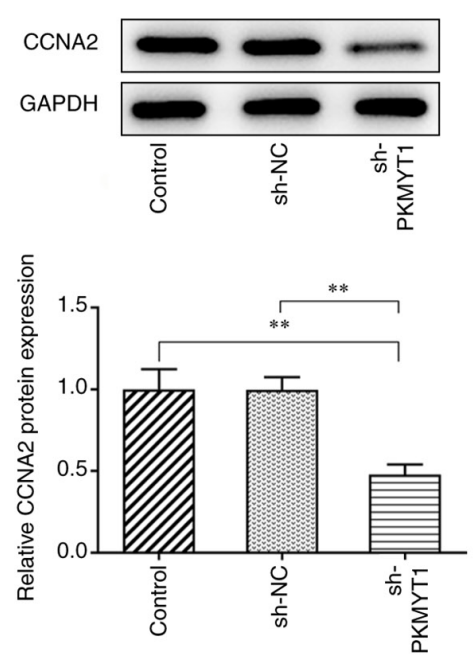

$\mathrm{H}$
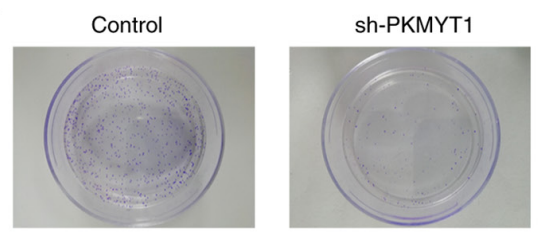

sh-PKMYT1+pcDNA

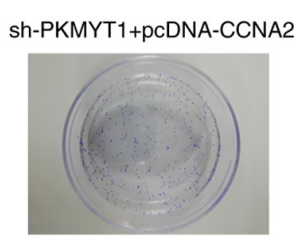

$\mathrm{F}$
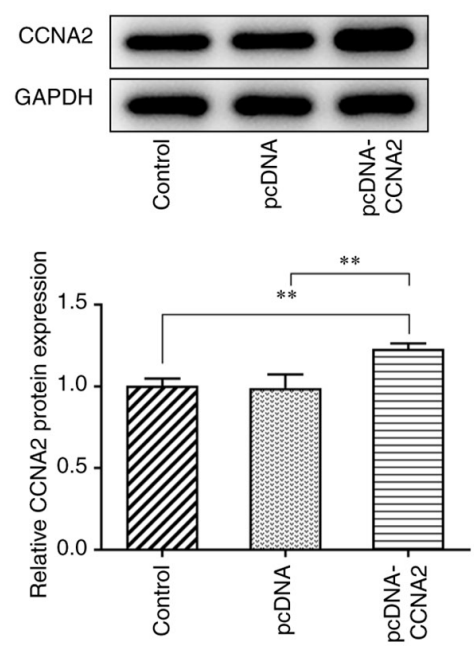
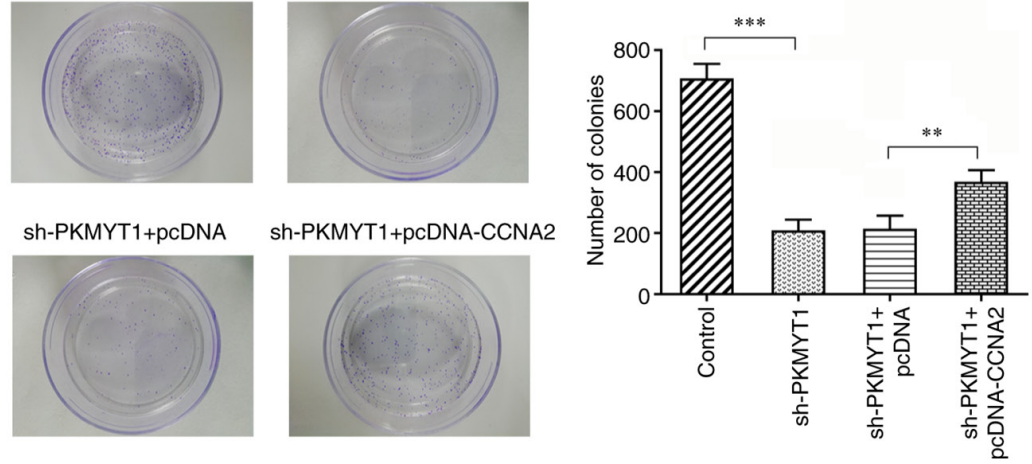

Figure 3. PKMYT1 knockdown inhibits the proliferation of SCC-9 cells by regulating CCNA2 expression. (A) PKMYT1 was found to be able to bind CCNA2 according to the STRING and GeneMANIA databases. (B and C) Relative mRNA and protein expression levels were detected using (B) RT-qPCR and (C) western blot analysis, respectively. (D) Co-IP assay was used for verification of the binding between PKMYT1 and CCNA2. (E) CCNA2 expression in the sh-PKMYT1 group was detected using western blot analysis. (F) CCNA2 expression in the pcDNA-CCNA2 group was detected using western blot analysis. (G) A Cell Counting Kit- 8 assay was used for the determination of cell proliferation. " $\mathrm{P}<0.05$ vs. sh-PKMYT1 + pcDNA. (H) A colony formation assay was used for the detection of cell colony formation. ${ }^{* *} \mathrm{P}<0.01,{ }^{* * * *} \mathrm{P}<0.001$. PKMYT1, protein kinase, membrane-associated tyrosine/threonine 1; CCNA2, cyclin A2; RT-qPCR, reverse transcription-quantitative PCR; co-IP, Co-immunoprecipitation; sh, short hairpin; sh-NC, negative control short hairpin RNA; WB, western blotting.

cells in the present study. This may be due to the relatively high level of keratin in CAL-27 cells, which is associated with cell proliferation and differentiation (23). The SCC-9 cell line has been used in the study of metastatic OSCC and displays strong migration and invasiveness (24). The present results also demonstrated that the proliferation and colony forming ability of SCC-9 cells were decreased following the suppression of PKMYT expression. Furthermore, PKMYT1 knockdown exerted inhibitory effects on cell invasion, migration and expression of migration-related proteins, including MMP2 and MMP9, as well as EMT-related proteins, including $\mathrm{N}$-cadherin, Snail and ZEB1. 
A

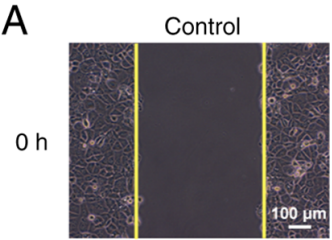

$48 \mathrm{~h}$

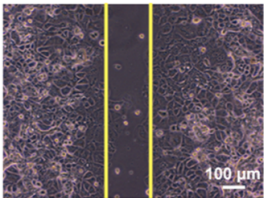

sh-PKMYT1
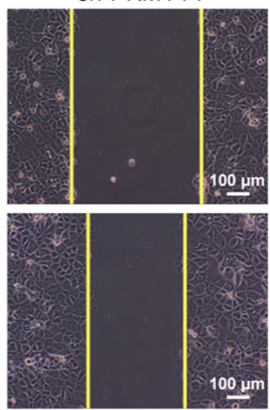

100 u

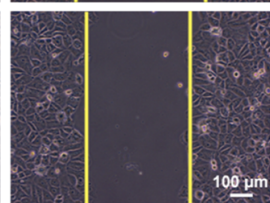

$100 \mathrm{p}$

B
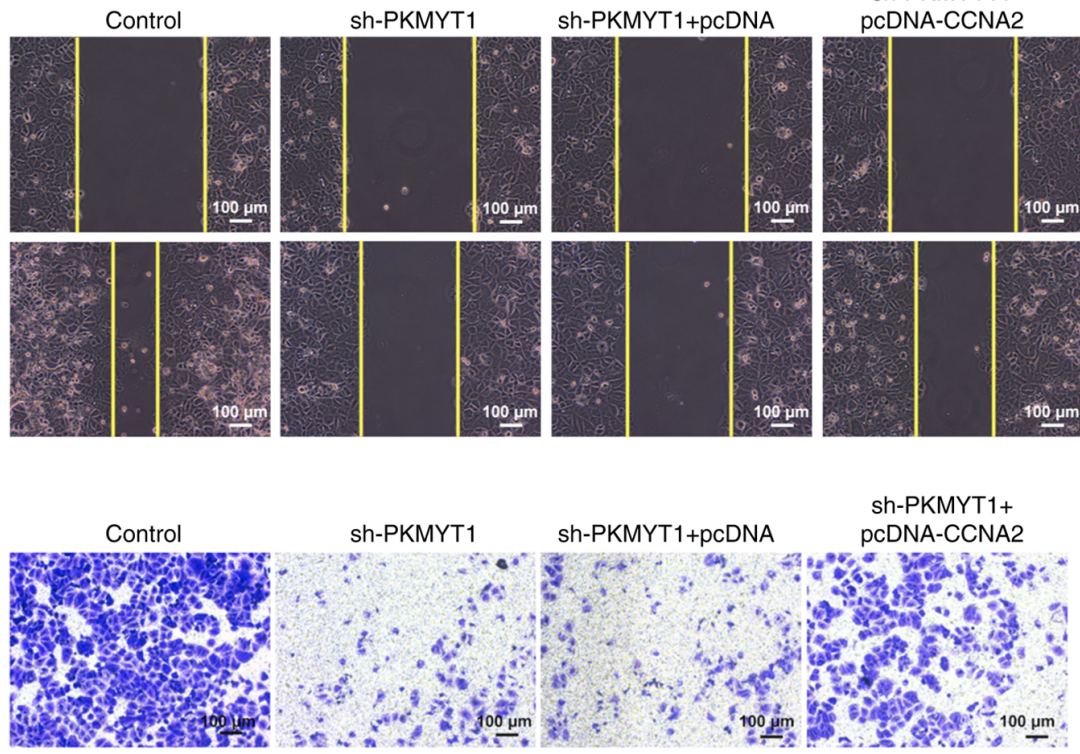

sh-PKMYT1
sh-PKMYT1+
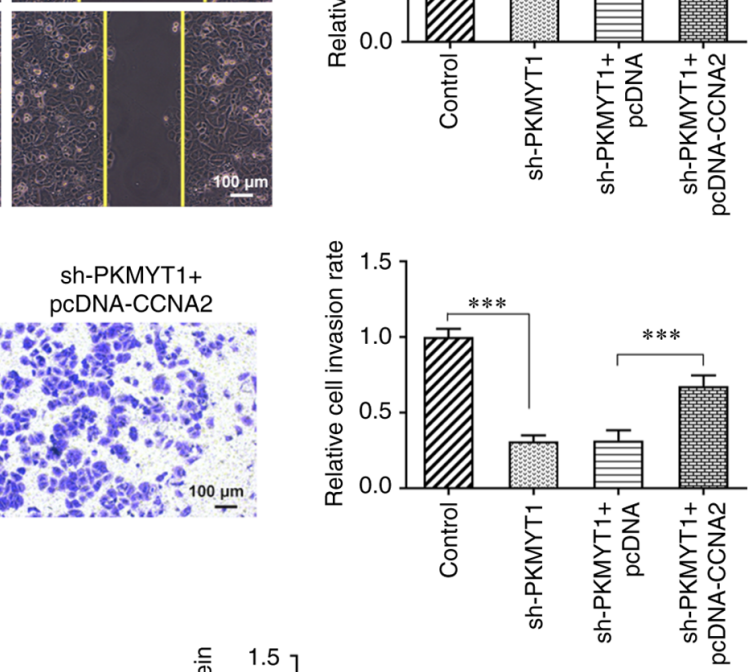

C
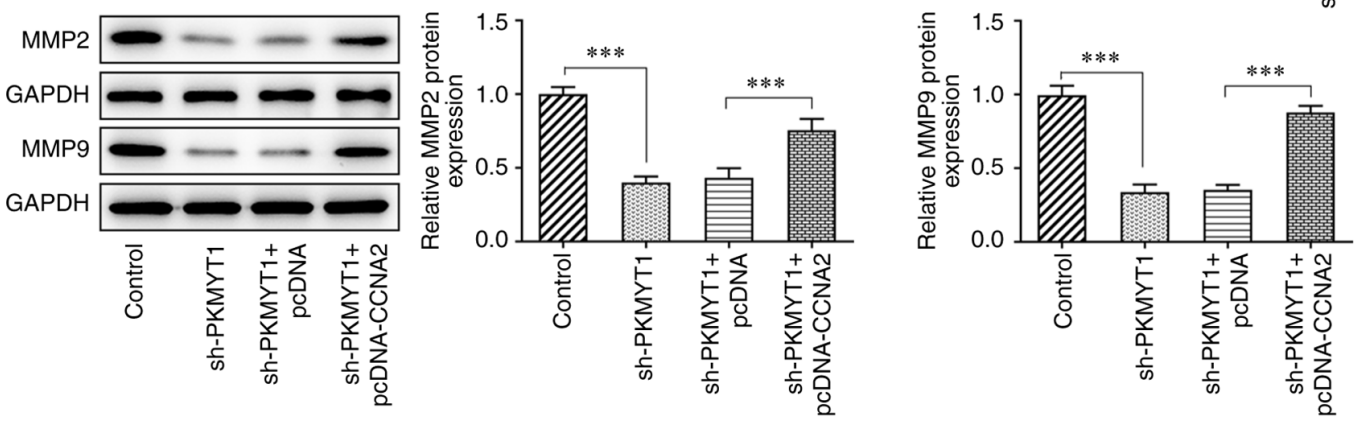

D
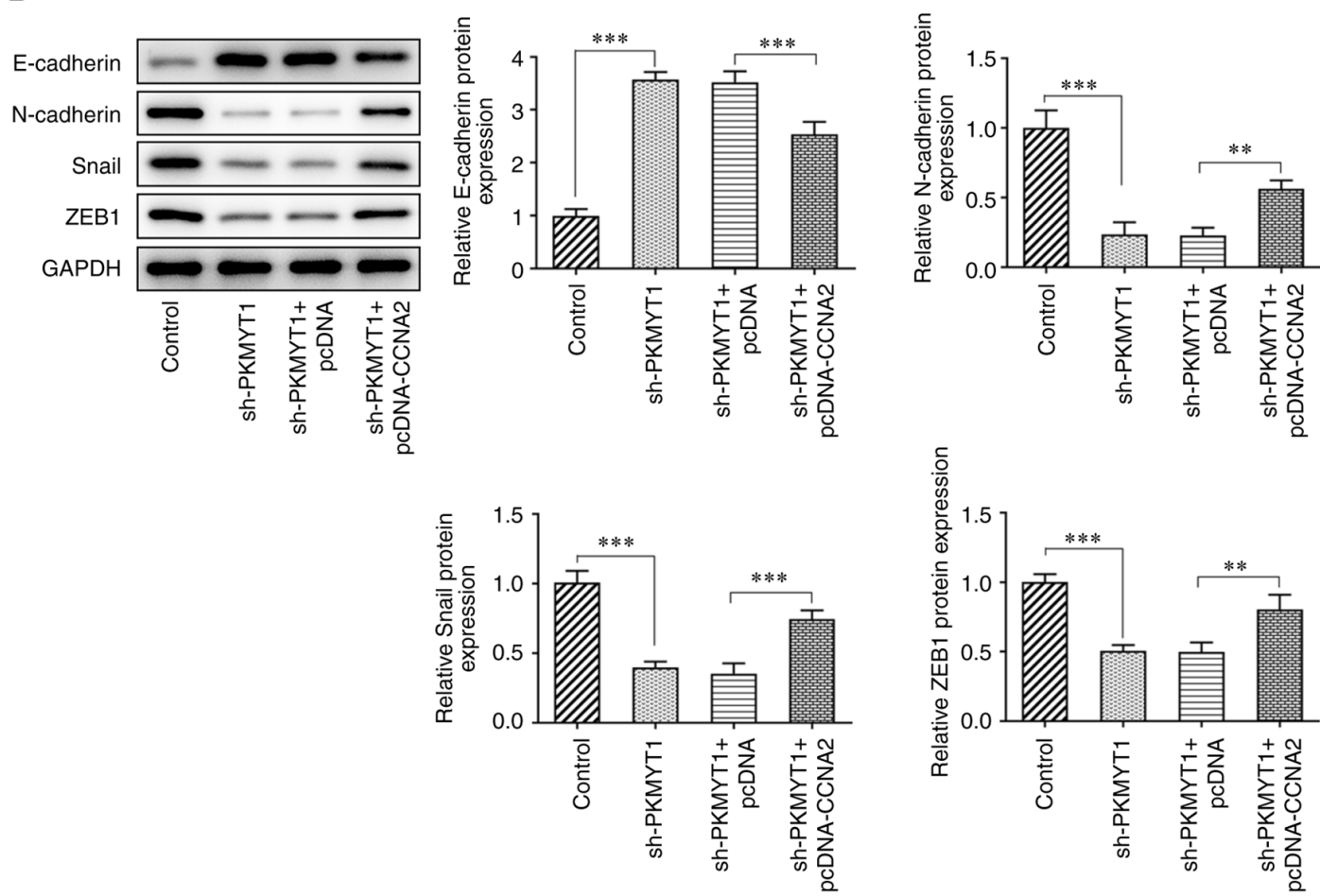

Figure 4. PKMYT1 knockdown inhibits the migration, invasion and EMT of SCC-9 cells by regulating CCNA2 expression. (A) Relative migration rate was detected by using a wound healing assay. (B) Relative cell invasion rate was detected by using a Transwell assay. (C) MMP2 and MMP9 expression was detected by using western blot analysis. (D) EMT-related protein expression was detected by using western blot analysis. ${ }^{* *} \mathrm{P}<0.01$, ${ }^{* * *} \mathrm{P}<0.001$. PKMYT1, protein kinase, membrane-associated tyrosine/threonine 1; EMT, epithelial-mesenchymal transition; CCNA2, cyclin A2; sh, short hairpin; ZEB1, zinc finger E-box binding homeobox 1. 
CCNA2 is a member of the highly conserved cyclin family, which participates in the regulation of $G_{1} / S$ and $G_{2} / M$ phases, and plays a role in DNA replication, transcription and tumor progression. CCNA2 has been reported to be highly expressed in a variety of cancer types (25). Yang et al (26) suggested that CCNA2 expression promotes tumor growth in hepatocarcinoma xenotransplantation mouse model. Furthermore, CCNA2 has also been previously revealed to participate in the EMT and metastasis of colorectal cancer (27). A previous study has demonstrated the presence of CCNA2, B1, D1 and E1, within the CCND1 gene, in 67 cases of primary OSCC (28). CCNA2 downregulation has also been revealed to regulate the migration and proliferation of trophoblasts (29). Additionally, CCND1 has been found to promote the invasion, migration and EMT of non-small cell lung carcinoma (NSCLC) cells, and may serve as a novel effective target for the treatment of NSCLC (30). CCNA2 regulates the EMT process probably by activating the transcription of EMT-related genes (such as Snail, Nanog and Myc) through the $\beta$-catenin (31) or Rho-associated coiled-coil containing protein kinase signaling pathways (32). According to the STRING and GeneMANIA databases, PKMYT1 may be associated with CCNA2. In the present study, it was demonstrated that PKMYT1 could bind to CCNA2 in SCC-9 cells, and its knockdown may inhibit the expression of CCNA2. Additionally, the decreased cell proliferation, migration, invasion and EMT caused by PKMYT1 knockdown were demonstrated to be reversed by CCNA2 overexpression, revealing that PKMYT1 may inhibit the malignant progression of OSCC via targeting CCNA2. However, the mechanism of CCNA2 downregulation remains to be further investigated to clarify whether it occurs specifically through regulating transcription, translation or promoting protein degradation. Furthermore, the present study is only preliminary and mainly based on one OSCC cell line. In future studies, clinical samples should be collected, and the clinical significance of PKMYT1 should be verified further.

In conclusion, the current study demonstrated that the expression of PKMYT1 and CCNA2 was upregulated in OSCC cell lines, and there was an association between the two proteins. Furthermore, it was revealed that PKMYT1 knockdown exerted inhibitory effects on cell proliferation, migration, invasion and EMT, while these effects were reversed by CCNA2 overexpression, revealing that PKMYT1 may serve a role in OSCC by targeting CCNA2. The findings of the present study may have revealed a novel biomarker or target that could be used in future treatment options for patients with OSCC.

\section{Acknowledgements}

Not applicable.

\section{Funding}

No funding was received.

\section{Availability of data and materials}

The datasets used and/or analyzed during the current study are available from the corresponding author on reasonable request.

\section{Authors' contributions}

YC made substantial contributions to the conception of the study and performed the experiments. WY was involved in performing the experiments and writing the manuscript. YC and WY confirm the authenticity of the raw data. Both authors have read and approved the final version of the manuscript.

\section{Ethics approval and consent to participate}

Not applicable.

\section{Patient consent for publication}

Not applicable.

\section{Competing interests}

The authors declare that they have no competing interests.

\section{References}

1. Bray F, Ferlay J, Soerjomataram I, Siegel RL, Torre LA and Jemal A: Global cancer statistics 2018: GLOBOCAN estimates of incidence and mortality worldwide for 36 cancers in 185 countries. CA Cancer J Clin 68: 394-424, 2018.

2. Panarese I, Aquino G, Ronchi A, Longo F, Montella M, Cozzolino I, Roccuzzo G, Colella G, Caraglia M and Franco R: Oral and Oropharyngeal squamous cell carcinoma: Prognostic and predictive parameters in the etiopathogenetic route. Expert Rev Anticancer Ther 19: 105-119, 2019.

3. Cohen EEW, Bell RB, Bifulco CB, Burtness B, Gillison ML, Harrington KJ, Le QT, Lee NY, Leidner R, Lewis RL, et al: The Society for immunotherapy of Cancer consensus statement on immunotherapy for the treatment of squamous cell carcinoma of the head and neck (HNSCC). J Immunother Cancer 7: 184, 2019.

4. Everts HB and Akuailou EN: Retinoids in cutaneous squamous cell carcinoma. Nutrients 13: 153, 2021.

5. Jia T, Wang F, Qiao B, Ren Y, Xing L, Zhang H and Li H: Knockdown of LncRNA PANDAR by CRISPR-dCas9 decreases proliferation and increases apoptosis in oral squamous cell carcinoma. Front Mol Biosci 8: 653787, 2021.

6. Lu Y, Zheng Z, Yuan Y, Pathak JL, Yang X, Wang L, Ye Z, Cho WC, Zeng $M$ and Wu L: The emerging role of exosomes in oral squamous cell carcinoma. Front Cell Dev Biol 9: 628103, 2021.

7. Glastonbury CM: Head and Neck Squamous Cell Cancer: Approach to Staging and Surveillance. In: Diseases of the Brain,Head and Neck, Spine 2020-2023: Diagnostic Imaging. Hodler J, Kubik-Huch RA and von Schulthess GK (eds). Springer, Cham, CH, 2020.

8. Cai L, Zhang X, Hou M and Gao F: Natural flavone tricetin suppresses oxidized LDL-induced endothelial inflammation mediated by Egr-1. Int Immunopharmacol 80: 106224, 2020.

9. Zhang Q, Zhao X, Zhang C, Wang W, Li F, Liu D, Wu K, Zhu D, Liu S, Shen C, et al: Overexpressed PKMYT1 promotes tumor progression and associates with poor survival in esophageal squamous cell carcinoma. Cancer Manag Res 11: 7813-7824, 2019.

10. Ghelli Luserna di Rorà A, Cerchione C, Martinelli G and Simonetti G: A WEE1 family business: Regulation of mitosis, cancer progression, and therapeutic target. J Hematol Oncol 13: $126,2020$.

11. Chen P, Zhang Z and Chen X: Overexpression of PKMYT1 facilitates tumor development and is correlated with poor prognosis in clear cell renal cell carcinoma. Med Sci Monit 26: e926755, 2020.

12. Liu Y, Qi J, Dou Z, Hu J, Lu L, Dai H, Wang H and Yang W: Systematic expression analysis of WEE family kinases reveals the importance of PKMYT1 in breast carcinogenesis. Cell Prolif 53: e12741, 2020.

13. Xuan ZH, Wang HP, Zhang XN, Chen ZX, Zhang HY and Gu MM: PKMYT1 aggravates the progression of ovarian cancer by targeting SIRT3. Eur Rev Med Pharmacol Sci 24: 5259-5266, 2020. 
14. Livak KJ and Schmittgen TD: Analysis of relative gene expression data using real-time quantitative PCR and the 2(-Delta Delta C(T)) method. Methods 25: 402-408, 2001.

15. von Mering C, Huynen M, Jaeggi D, Schmidt S, Bork P and Snel B: STRING: A database of predicted functional associations between proteins. Nucleic Acids Res 31: 258-261, 2003.

16. Warde-Farley D, Donaldson SL, Comes O, Zuberi K, Badrawi R, Chao P, Franz M, Grouios C, Kazi F, Lopes CT, et al: The GeneMANIA prediction server: Biological network integration for gene prioritization and predicting gene function. Nucleic Acids Res 38: W214-W220, 2010.

17. Yan X and Su H: YM155 Down-Regulates Survivin and induces P53 Up-regulated modulator of apoptosis (PUMA)-dependent in oral squamous cell carcinoma cells. Med Sci Monit 23: 1963-1972, 2017.

18. Long HP, Liu JQ, Yu YY, Qiao Q and Li G: PKMYT1 as a potential target to improve the radiosensitivity of lung adenocarcinoma. Front Genet 11: 376, 2020.

19. Wang J, Wang L, Chen S, Peng H, Xiao L, E Du, Liu Y, Lin D, Wang Y, Xu Y and Yang K: PKMYT1 is associated with prostate cancer malignancy and may serve as a therapeutic target. Gene 744: 144608, 2020.

20. Jeong D, Kim H, Kim D, Ban S, Oh S, Ji S, Kang D, Lee H, Ahn TS, Kim HJ, et al: Protein kinase, membrane-associated tyrosine/threonine 1 is associated with the progression of colorectal cancer. Oncol Rep 39: 2829-2836, 2018.

21. Yadav M, Pradhan D and Singh RP: Integrated analysis and identification of nine-gene signature associated to oral squamous cell carcinoma pathogenesis. 3 Biotech 11: 215, 2021.

22. Sumita Y, Yamazaki M, Maruyama S, Abé T, Cheng J, Takagi R and Tanuma JI: Cytoplasmic expression of SOX9 as a poor prognostic factor for oral squamous cell carcinoma. Oncol Rep 40: 2487-2496, 2018.

23. Jung HM, Phillips BL, Patel RS, Cohen DM, Jakymiw A, Kong WW, Cheng JQ and Chan EK: Keratinization-associated miR-7 and miR-21 regulate tumor suppressor reversion-inducing cysteine-rich protein with kazal motifs (RECK) in oral cancer. J Biol Chem 287: 29261-29272, 2012.

24. Chou SC, Azuma Y, Varia MA and Raleigh JA: Evidence that involucrin, a marker for differentiation, is oxygen regulated in human squamous cell carcinomas. Br J Cancer 90: 728-735, 2004.
25. Gao T, Han Y, Yu L, Ao S, Li Z and Ji J: CCNA2 is a prognostic biomarker for ER+ breast cancer and tamoxifen resistance. PLoS One 9: e91771, 2014

26. Yang F, Gong J, Wang G, Chen P, Yang L and Wang Z: Waltonitone inhibits proliferation of hepatoma cells and tumorigenesis via FXR-miR-22-CCNA2 signaling pathway. Oncotarget 7: 75165-75175, 2016.

27. Bendris N, Arsic N, Lemmers B and Blanchard JM: Cyclin A2, Rho GTPases and EMT. Small GTPases 3: 225-228, 2012.

28. Monteiro LS, Diniz-Freitas M, Warnakulasuriya S, Garcia-Caballero T, Forteza-Vila J and Fraga M: Prognostic significance of Cyclins A2, B1, D1, and E1 and CCND1 numerical aberrations in oral squamous cell carcinomas. Anal Cell Pathol (Amst) 2018: 7253510, 2018.

29. Li X, Ma XL, Tian FJ, Wu F, Zhang J, Zeng WH, Lin Y and Zhang Y: Downregulation of CCNA2 disturbs trophoblast migration, proliferation, and apoptosis during the pathogenesis of recurrent miscarriage. Am J Reprod Immunol 82: e13144, 2019.

30. Ruan JS, Zhou H, Yang L, Wang L, Jiang ZS and Wang SM: CCNA2 facilitates epithelial-to-mesenchymal transition via the integrin $\alpha v \beta 3$ signaling in NSCLC. Int J Clin Exp Pathol 10: 8324-8333, 2017.

31. Cheung CT, Bendris N, Paul C, Hamieh A, Anouar Y, Hahne M, Blanchard JM and Lemmers B: Cyclin A2 modulates EMT via $\beta$-catenin and phospholipase $\mathrm{C}$ pathways. Carcinogenesis 36 : 914-924, 2015.

32. Li J, Ying Y, Xie H, Jin K, Yan H, Wang S, Xu M, Xu X, Wang X, Yang $\mathrm{K}$, et al: Dual regulatory role of CCNA2 in modulating CDK6 and MET-mediated cell-cycle pathway and EMT progression is blocked by miR-381-3p in bladder cancer. FASEB 33: 1374-1388, 2019.

(i) (5) This work is licensed under a Creative Commons Attribution-NonCommercial-NoDerivatives 4.0 International (CC BY-NC-ND 4.0) License. 